\title{
Multimodal characterization of the visual network in Huntington's disease gene carriers
}

Sarah Gregorya*, Omar F F Odish ${ }^{\mathrm{b}^{*}}$, Isabella Mayer ${ }^{\mathrm{c}^{*}}$, James Mills ${ }^{\mathrm{d}}$, Eileanoir B Johnsonª, Rachael I Scahilla, John Rothwelle, Geraint Reesf, Jeffrey D Longd,g, Sarah J Tabrizia, Raymund A C Roos ${ }^{\mathrm{h}}$, Michael Orth ${ }^{\mathrm{c}}$

*equal contribution

a Huntington's Disease Centre, UCL Institute of Neurology, London, UK

${ }^{b}$ Department of Neurology, University Medical Center Groningen, Groningen, The Netherlands

cDepartment of Neurology, Ulm University Hospital, Ulm, Germany

${ }^{d}$ Department of Psychiatry, University of Iowa, Iowa City, IA, USA

eSobell Department of Motor Neuroscience and Movement Disorders, University College London

Institute of Neurology, Queen Square, London, UK

fWellcome Trust Centre for Neuroimaging, University College London, London, UK

gDepartment of Biostatistics, University of Iowa, Iowa City, IA, USA

${ }^{h}$ Department of Neurology, Leiden University Medical Centre, Leiden, The Netherlands

\section{Contact for correspondence}

Michael Orth, M.D., Ph.D.

Department of Neurology, Ulm University Hospital

Oberer Eselsberg 45/1

89081 Ulm, Germany

e-mail: michael.orth@uni-ulm.de

Tel: +49-731 50063095; Fax: +49-73150063082 


\section{Highlights}

- Structural integrity relates to efficient functional processing within the visual system

- More white matter disorganization and slower, more disperse, signal transmission in Huntington's

- Dissociation in Huntington's between normal behaviour and abnormal visual network function 


\section{Abstract}

Objective: A sensorimotor network structural phenotype predicted motor task performance in a previous study in Huntington's disease (HD) gene carriers. We investigated in the visual network whether structure - function - behaviour relationship patterns, and the effects of the HD mutation, extended beyond the sensorimotor network.

Methods: We used multimodal visual network MRI structural measures (cortical thickness and white matter connectivity), plus visual evoked potentials and task performance (Map Search; Symbol Digit Modalities Test) in healthy controls and HD gene carriers.

Results: Using principal component (PC) analysis, we identified a structure - function relationship common to both groups. PC scores differed between groups indicating white matter disorganization (higher RD, lower FA) and slower, and more disperse, VEP signal transmission (higher VEP P100 latency and lower VEP P100 amplitude) in HD than controls while task performance was similar.

Conclusions: HD may be associated with reduced white matter organization and efficient visual network function but normal task performance.

Significance: These findings indicate that structure - function relationships in the visual network, and the effects of the HD mutation, share some commonalities with those in the sensorimotor network. However, implications for task performance differ between the two networks suggesting the influence of network specific factors.

Key words: structural MRI; tractography; visual evoked potentials; principal component analysis 


\section{Introduction}

Brain structure, function and behaviour are clearly linked, but the relationship between them is highly complex. In Huntington's disease (HD), for example, cerebral white and grey matter loss, particularly within the basal ganglia is detectable from 15-20 years prior to clinical diagnosis (Thieben et al. 2002; Aylward 2007; Rosas et al. 2008; Georgiou-Karistianis et al. 2013), yet performance levels remain normal for a considerable time suggesting onset of compensatory processes (Tabrizi et al. 2013; Kloppel et al. 2015; Gregory et al. 2018b). On the other hand, structural imaging measures of brain volume, together with task performance can improve predictions of motor diagnosis based solely on the HTT mutation and age (Tabrizi et al. 2013). Together, this supports the notion that structural alterations contribute to functional brain changes underlying the manifestation of clinical signs of HD.

Examining the relationship between structure, function and task performance within $a$ priori selected brain networks in HD gene carriers could help differentiate between a) network-wide changes that are specific to the presence of the HTT mutation and b) natural variations in network properties amongst healthy people that influence the effects of the HTT mutation. We previously examined this concept within the sensorimotor network in HD using multimodal structural and functional data (Orth et al. 2016). We found a structural pattern of reduced volume and cortical thickness in sensorimotor regions coupled with increased diffusivity in white matter pathways that was closely linked to the HD mutation and predicted performance. However, we also identified an inverse relationship between axial diffusivity (AD; diffusivity in the main direction of the fibre) and radial diffusivity (RD; diffusivity perpendicular to the main fibre) that was common to both controls and HD gene carriers. This relationship pattern predicted HD disease status and motor performance independent of HD-associated 
factors such as CAG, age and brain volume. This relationship may, therefore, reflect a pattern of natural variability in white matter microstructure that itself does not cause disease. However, in the presence of the HD mutation it may modify the effects of HD pathogenesis on white matter microstructure.

Given our previous findings, here we asked to what extent these observations were specific to the sensorimotor network or whether they reflected patterns that are also present in other networks potentially impacted by HD pathology. This has important implications for disease modification in terms of network-wide versus network-specific patterns of structure, function and behaviour relationships. Although characteristically defined by motor, cognitive and neuropsychiatric symptoms, the visual cortex is one of the first areas affected in HD with evidence of neuronal loss (Rosas et al. 2008; Tabrizi et al. 2009, 2012; Wolf et al. 2014; Rub et al. 2015), white matter pathway degeneration (McColgan et al. 2018) and deficits in visual-processing (Rosas et al. 2008; Scahill et al. 2013; Tabrizi et al. 2013; Paulsen et al. 2014; Johnson et al. 2015).

Consistent with our earlier study, we used multimodal MRI and electrophysiological data to examine the relationship between structural integrity, functional processing and task performance in the visual network in a cohort of controls and HD gene carriers. We investigated both structure (V1 cortical thickness; visual pathway connectivity) and function (Visual Evoked Potentials (VEP)) in conjunction with task performance on Map Search and Symbol Digit Modalities Test (SDMT). We investigated correlations between individual measures and then used Principal Component Analysis (PCA) to identify patterns across modalities. Finally, we examined to what extent these patterns identified group status. We predicted independent structure-function relationships a) 
characteristic of HD only and b) common to both controls and HD reflecting natural variability.

\section{Methods}

\subsection{Participants}

Participants were recruited from the Leiden site of the international multi-site Track-On HD study (Orth et al. 2016) and comprised 20 HD gene mutation carriers (mean age 49.2 years, 12 female) and 24 healthy controls (mean age 52.5 years, 16 female). All HD gene mutation carriers had a CAG repeat length $\geq 40$; and a burden of pathology score (disease burden) greater than 250 ((CAG repeat length - 35.5) x age) (Penney et al. 1997; Tabrizi et al. 2009) (Table 1). Healthy family members without the HD mutation or partners were recruited as control participants. All participants were screened for major psychiatric, neurological or medical disorders or a history of severe head injury. Education was measured using the International Standard Classification of Education (ISCED) that distinguishes 10 different levels of education. The total motor score was obtained from the motor part of the Unified Huntington's Disease Rating Scale (UHDRS). Visual acuity was documented prior to VEP acquisition, and all participants had normal or corrected-to-normal vision. The study was approved by the Leiden University Institutional Review Board. All participants gave their written informed consent to the study, and all methods were used and experiments performed, in accordance with the ethical standards laid down in the 1964 Declaration of Helsinki and its later amendments.

\subsection{Behavioural Measures}


The Map Search Task is a subtest from the Test of Everyday Attention and measures visuospatial selective attention (Murray and Stout 2014). Participants were presented with an A3 sized map, which displayed a portion of the city of Philadelphia in the United States. They were then timed for two minutes while they searched for and circled a target symbol that occurred in multiple places on the map among other distracter symbols. After one minute, the examiner exchanged the pen for a different color to facilitate differentiation of those responses made in the first and second minutes of testing. Test performance was measured as the number of correctly circled target symbols, scored separately at one minute and at two minutes with a maximum possible score of 80. For the current study, we used the percentage number of correct responses in two minutes as a behavioural performance measure.

The Symbol Digit Modalities Test (SDMT) is a test of visuomotor integration, measuring visual attention and motor speed. Participants were required to match symbols and digits as quickly as possible, following a key located at the top of the page during a 90 second period; the total number of correct responses were recorded and included as our second behavioural performance measure.

\subsection{Electrophysiology Measures}

Four Ag-AgCl-electrodes were attached to the scalp at position 01, 02 and $\mathrm{Oz}$ with $\mathrm{Cz}$ as a reference according to the international 10-20-system for electrode placement. Participants were seated at a distance of $1 \mathrm{~m}$ in front of a 23 -inch computer screen displaying a checkerboard pattern that filled the entire screen with squares of $1^{\circ}$ visual angle flashing at a frequency of $2 \mathrm{~Hz}$. The brightness of the squares was $100 \mathrm{lux}$ with a black/white contrast. For each eye, 2 x 100 trials were recorded. The duration of each trial was dependent on the registered signals, approximately one minute in duration, or 
30 seconds per trial. The stimulus was presented continuously and flashing, with no stop between trials. VEP’s were obtained using Medelec Synergy version 11.0 (Oxford Instruments, Abingdon, United Kingdom). Data were filtered and visually checked for artefacts; noisy trials were deleted from the set. The trials were averaged and peak latencies and peak-to-peak amplitudes of N70, P100 and N135 were identified. The N70 was defined as the most prominent negative peak between 60 and $80 \mathrm{~ms}$ post stimulus. For the P100 a time window of $90-115 \mathrm{~ms}$ and for the N135 a time window of 115150ms was applied.

\subsection{MRI Measures}

\subsubsection{Cortical Thickness}

3D T1 images were acquired as described (Tabrizi et al. 2013). Cortical thickness measures were generated for each participant using FreeSurfer version 5.3.0 applying default parameters and optimized for 3T data (Fischl and Dale 2000). Measures were extracted from Brodmann area in the left hemisphere: BA17 (Primary Visual Cortex) https://surfer.nmr.mgh.harvard.edu/fswiki/BrodmannAreaMaps. All segmentations were visually inspected for accuracy, blind to participant status.

\subsubsection{Diffusion Tensor Imaging}

Diffusion-weighted images with 42 unique gradient directions $\left(b=1000 \mathrm{sec} / \mathrm{mm}^{2}\right)$ and one image with no diffusion weighting $\left(b=0 \mathrm{sec} / \mathrm{mm}^{2}\right)$ were acquired using a Phillips Achieva scanner. Acquisition parameters were as follows: $\mathrm{TE}=56 \mathrm{~ms}$ and $\mathrm{TR}=11 \mathrm{~s}$, with voxel size $1.96 \times 1.96 \times 2 ; 75$ slices were collected for each diffusion-weighted and nondiffusion weighted (B0) volume. The diffusion data were preprocessed using standard FSL pipelines (Smith et al. 2004). 
Data were initially quality checked for movement artefacts and then corrected for eddy current distortions. Diffusion tensors were fitted to the corrected data using DTIFit; FA (fractional anisotropy), AD (axial diffusivity) and RD (radial diffusivity) values were subsequently derived from the tensors. The B0 image and the T1-weighted structural image were both skull-stripped using the Brain Extraction Tool and then manually edited. The T1 image was then registered to the B0 image using FLIRT (Jenkinson and Smith 2001). Crossing fibres were modelled using Bedpostx (Behrens et al. 2007). Probtrackx was used for fibre-tracking of the visual pathway using three regions of interest: the primary visual cortex (V1), extrastriate area V4 and the visual thalamus (Behrens et al. 2003). All seed regions were created in standard space using the Anatomy toolbox and then warped into native space (using the DARTEL inverse deformation parameters; see resting state fMRI methods section) for fibre-tracking. Masks were used to exclude streamlines that tracked into the right hemisphere and into grey matter, cerebrospinal fluid (CSF) or dura. The visual pathway images were then warped into diffusion space using FLIRT and FA, AD and RD values extracted for each participant.

\subsection{Statistical Analyses}

Control and HD groups were compared for each individual modality using two sample ttests with FDR adjustment for multiple comparisons. The equality of variances assumption was tested and the Satterthwaite approximation of the standard errors and degrees of freedom were used when necessary.

Pearson's correlations were performed between the structure-function and behavioural measures across a) HD gene carriers participants and b) control participants . Structural integrity and functional processing measures were investigated through PCA, a method 
used to reduce the dimensionality of multivariate data by producing linear combinations of the original variables. These principal components (PC) are mutually independent and retain most of the variability present in the original measures (Jolliffe 2002). The number of components was determined from the results of 2-fold split-sample validation (Eastment and Krzanowski 1982).

After obtaining the PCs, a series of ANCOVA models, adjusting for age and gender, were utilized to evaluate the relationships among the PCs, the behavioural performance measures, and group status. The first set of models was used to examine the relationship of the PCs and the behavioural performance measures with group status. The next set assessed the association of the PCs and the behavioural performance measures (1) without controlling for group status, (2) controlling for group status, and (3) with a group*component interaction. Corrections for multiple comparisons were made using an FDR threshold of $q=0.05$ within all sets of analyses (Benjamini and Hochberg 1995).

\section{Results}

\subsection{Individual Modality Analyses}

Demographic and clinical data for the control and HD groups are presented in Table 1. We focused on eight variables that captured the structure - function relationship within the visual system in the left hemisphere. These included 4 structural MRI measures (V1 cortical thickness, FA, RD, AD) and 2 electrophysiological measures (VEP P100 latency and amplitude recorded from 01) in addition to 2 behavioural performance measures (Map Search and SDMT). We first compared each individual modality in controls and the HD group (Table 2). Group comparisons revealed evidence of significantly higher RD $(p=0.014, q=0.11)$ in the visual pathway connecting the visual thalamic region and $\mathrm{V} 1$ and non-significantly longer VEP P100 latencies $(p=0.0816, q=0.24)$ for those with HD 
versus controls. In contrast, V1 cortical thickness, FA, AD, VEP P100 amplitude and both behavioural measure were similar in the HD group and the controls. Note that the Satterthwaite approximations for standard errors and degrees of freedom were used for DTI FA, $A D$, and RD because of unequal variances between the groups for these measures.

\section{Multimodal Structure-Function-Behaviour Analyses}

Correlations were performed between the structure-function and behavioural measures across a) HD gene carriers (Table 3) and b) control participants (Table 4). Across HD participants, worse SDMT performance was associated with lower fractional anisotropy $(\mathrm{r}=0.63, \mathrm{p}=0.0091, \mathrm{q}=0.055)$, higher radial diffusivity $(\mathrm{r}=-0.55, \mathrm{p}=0.026, \mathrm{q}=0.11)$ and longer VEP P100 latency ( $r=-0.41, \mathrm{p}=0.079, \mathrm{q}=0.24)$; worse Map Search performance also correlated with longer VEP P100 latency ( $r=-0.60, p=0.0056, q=0.055)$ (Table 3). Correlations for control participants, on the other hand, were not significantly different from zero (Table 4).. We then used a regression model to further investigate the group factor. Before FDR adjustment, we find marginal significance between the groups in the association of SDMT with DTI FA, DTI RD, and VEP P100 Amplitude ( $p=0.063,0.061$, and 0.044 respectively) and in the association of Map Search with VEP P100 Latency $(\mathrm{p}=0.060)$. The FDR adjusted values are all non-significant $(\mathrm{q}>0.15$ for all).

Next, we employed PCA to describe the patterns of relationships within the dimensions of the structure-function measures (Figure 1; Table 5). The PCA was run on a reduced number of 36 participants who had complete data (20 controls and 16 HD). The six structure-function measures and their relationships could be reduced to 3 principal components (Figure 1 as determined by 2-fold cross validation. The 3 components explained $74.5 \%$ of the variance. The first PC (PC1) explained $34.0 \%$ of data variance 
and included correlations between increased FA, lower RD in the visual pathway connecting the visual thalamic region and V1, thicker V1, reduced VEP P100 latency and increased VEP P100 amplitude (Figure 1A). PC1 scores were associated with group status, differentiating between controls and HD participants $(\mathrm{t}=-2.34, \mathrm{p}=0.026, \mathrm{q}=0.077)$. The controls showed mean PC scores of 0.286 (4 of 20 had negative scores) compared to the HD group, in which the majority of participants (11 of 16) had negative scores with an average negative PC score of -0.474 (Figure 1B). This is indicative of higher RD, lower FA, higher VEP P100 latency and lower VEP P100 amplitude in the HD group compared to the control group.

The second PC (PC2; 23.6\% of variance explained) showed a pattern of reduced FA and $\mathrm{AD}$ in the visual pathway connecting the visual thalamic region and $\mathrm{V} 1$, coupled with an increase in V1 cortical thickness (Figure 1A). The third PC (PC3; 16.9\% of variance explained) captured a pattern of reduced AD in the visual pathway connecting the visual thalamic region and reduced V1 cortical thickness (Figure 1A). Neither PC2 $(t=-0.23$, $\mathrm{p}=0.82, \mathrm{q}=0.82)$ nor PC3 $(\mathrm{t}=-0.66, \mathrm{p}=0.51, \mathrm{q}=0.77)$ were associated with group status (Figure 1C and D).

\section{Discussion}

In this study, we have identified structure-function relationships showing an association between structural integrity and efficient functional processing within the visual system in healthy controls and HD. Lower levels of white matter organization and VEP responsivity correlated with lower levels of task performance in HD gene carriers, but not for control participants. We further examined these associations using Principal Component Analysis, identifying a structure-function relationship of white matter organization and VEP responsivity. Although this relationship was common to both HD 
and controls, the majority of HD gene carriers displayed negative scores, such that they were characterized by increased white matter disorganization in the visual pathway and a less effective visual processing system. However, despite this disrupted structurefunction relationship, HD gene carriers performed visual tasks at a normal level. Given the correlations between structure-function measures and performance, this would indicate that despite an abnormal structure-function relationship, HD gene carriers may experience some degree of compensatory brain activity in the visual network.

We previously explored structure-function relationships within the sensorimotor network in HD and characterised a macro- and micro-structural phenotype associated with HD (Orth et al. 2016). We showed that structural degeneration within the sensorimotor network was related to both motor performance and pathology, but we also identified an independent inverse relationship between axial and radial diffusivity that was common to both HD and control groups and which predicted motor performance and disease status. Here, we have similarly identified a white-matter structural pattern in the visual network common to both controls and HD gene carriers, but which were also associated with visual processing.

Using principal component analysis, we showed that controls and HD gene carriers shared a similar structure-function relationship of higher white matter organization (i.e. higher FA and lower RD) combined with higher VEP responsivity (i.e. higher amplitude and lower latency) and to a lesser extent higher cortical thickness in the V1. However, despite the fact that this relationship was common to both groups and, therefore, likely due to natural biological variation in these network properties in the population, it actually differentiated the control and HD gene carrier groups, i.e. the average PC scores for each group differed significantly. As such, the majority of the HD group displayed negative scores, exhibiting a converse pattern of reduced white matter organization (i.e. 
lower FA and higher RD) and VEP responsivity (i.e. lower amplitude and higher latency). This supports our previous findings whereby we identified a pattern of volume loss and increased diffusivity in the sensorimotor network, associated with HD pathology. In healthy people the structure - function relationship may be variable, something we found in the somatosensory and now in the visual network so that in some people white matter organization will be higher, and function better, than in others. The HD mutation may exert its effect on top of that normal variability, and it is conceivable that these effects may take longer in a person with, by nature, higher than in someone with lower white matter organization. Given that we find evidence to support this notion now in two networks it may be worth extending this to other networks, e.g. those involved in cognition (Gregory et al. 2018a).

While we have not examined clinical markers of HD in the current study, the effect of HD pathology on the efficiency of the visual network is evident and reflects a pattern not only of structural disturbance as was the case in the sensorimotor network, but also of functional impairment. Despite this structural degeneration and functional deficit, there were no significant behavioural differences in visual task performance between controls and HD gene carriers at a group level. However, in the HD group the results of the correlation analyses between measures of structure-function (i.e. diffusivity and VEP responsivity) and behaviour, had shown that higher FA and reduced RD both tended to be correlated with improved SDMT performance, while shorter VEP latency tended to be associated with both better SDMT and Map Search performance. PCA analyses further revealed relationships between structural and functional network properties that vary systematically between individuals including HD gene carriers. However, although HD pathology additionally affects network properties of efficient visual processing and associated structure, there was no evidence of abnormal task performance at the group 
level. The correlations between behaviour, which is unimpaired, and higher levels of white matter organization and VEP responsivity may, therefore, indicate some degree of compensatory brain activity.

The two remaining components from our PCA analysis display patterns that are common to both controls and HD, but are not related to pathology, i.e. they did not distinguish between groups. The second component shows a pattern of reduced FA, reduced $\mathrm{AD}$ and increased cortical thickness, while the third shows a pattern of reduced $\mathrm{AD}$ and reduced cortical thickness. Interestingly in both the second and third components, there is an inverse relationship between $\mathrm{AD}$ and (lower levels of) RD, similar to that within the sensorimotor network - this was also independent of group status. The underlying basis of reduced FA and AD in terms of white matter organization is unclear, but reduced FA may be associated with increased RD. This may also explain why FA reductions are substantially pronounced as part of component three, because here RD is considerably lower.

In summary, we have identified patterns of visual network white matter organization that were correlated with both visual processing and visual performance. Interestingly, the pattern of higher white matter organization and visual processing efficiency, while common to both control and HD gene carriers, distinguished the groups describing higher levels of white matter disorganization and impaired visual processing in HD. In common with our previous analysis of the sensorimotor network we also characterized inverse patterns of $\mathrm{AD}$ and $\mathrm{RD}$ in the visual network; however, in the sensorimotor network we had not seen a functional contribution as we did here in the visual network (Orth et al. 2016). Our findings indicate that the structure-function relationships, and the susceptibility to the effects of the HTT mutation, may differ between brain networks 
in HD. This requires further investigation across a series of other networks, which may be particularly relevant and/or susceptible to the effects of HD pathology.

\section{Acknowledgements}

The authors thank the Track-On study participants and their families. CHDI/ High Q Foundation, a not-for-profit organization dedicated to finding treatments for HD, funded the study but had no role in the collection, analysis and interpretation of data and in the writing of the manuscript, or the decision to publish the findings. S.G., R.S., G.R., E.J. and S.T. receive support from a Wellcome Trust Collaborative Award $(200181 / \mathrm{Z} / 15 / \mathrm{Z})$

\section{Competing financial interests}

The authors declare no competing financial interests.

\section{Track-On investigators:}

M Jacobs, A Schoonderbeek (Leiden University Medical Centre, Leiden); J Stout (Monash University, Australia); BR Leavitt (University of British Columbia, Vancouver, Canada); A Durr (ICM Institute, Paris); R Reilmann (George Huntington Institut, Germany); Gail Owen, Adeel Razi, (University College London, London, UK); David Craufurd (University 
of Manchester, UK); D Langbehn (University of Iowa, Iowa, USA); G B Landwehrmeyer (University of Ulm, Germany).

\section{References}

Aylward EH. Change in MRI striatal volumes as a biomarker in preclinical Huntington's disease. Brain Res Bull [Internet]. 2007;72:152-8. Available from: http://www.ncbi.nlm.nih.gov/pubmed/17352939

Behrens TE, Berg HJ, Jbabdi S, Rushworth MF, Woolrich MW. Probabilistic diffusion tractography with multiple fibre orientations: What can we gain? Neuroimage [Internet]. 2007;34:144-55. Available from: http://www.ncbi.nlm.nih.gov/pubmed/17070705

Behrens TE, Woolrich MW, Jenkinson M, Johansen-Berg H, Nunes RG, Clare S, et al. Characterization and propagation of uncertainty in diffusion-weighted MR imaging. Magn Reson Med [Internet]. 2003;50:1077-88. Available from:

http://www.ncbi.nlm.nih.gov/pubmed/14587019

Benjamini Y, Hochberg Y. Controlling the False Discovery Rate - a Practical and Powerful Approach to Multiple Testing. J R Stat Soc Ser B-Methodological. 1995;57:289-300. Eastment HT, Krzanowski WJ. Cross-validatory choice of the number of components from a principal component analysis. Technometrics. 1982; 
Fischl B, Dale AM. Measuring the thickness of the human cerebral cortex from magnetic resonance images. Proc Natl Acad Sci U S A [Internet]. 2000;97:11050-5. Available from: http://www.ncbi.nlm.nih.gov/pubmed/10984517

Georgiou-Karistianis N, Poudel GR, Dominguez DJ, Langmaid R, Gray MA, Churchyard A, et al. Functional and connectivity changes during working memory in Huntington's disease: 18 month longitudinal data from the IMAGE-HD study. Brain Cogn [Internet]. 2013;83:80-91. Available from: http://www.ncbi.nlm.nih.gov/pubmed/23938592

Gregory S, Crawford H, Seunarine K, Leavitt B, Durr A, Roos RAC, et al. Natural biological variation of white matter microstructure is accentuated in Huntington's disease. Hum Brain Mapp. 2018a;.

Gregory S, Long JD, Klöppel S, Razi A, Scheller E, Minkova L, et al. Testing a longitudinal compensation model in premanifest Huntington's disease. Brain. 2018b;.

Jenkinson M, Smith S. A global optimisation method for robust affine registration of brain images. Med Image Anal [Internet]. 2001;5:143-56. Available from: http://www.ncbi.nlm.nih.gov/pubmed/11516708

Johnson EB, Rees EM, Labuschagne I, Durr A, Leavitt BR, Roos RA, et al. The impact of occipital lobe cortical thickness on cognitive task performance: An investigation in Huntington's Disease. Neuropsychologia [Internet]. 2015;79:138-46. Available from: http://www.ncbi.nlm.nih.gov/pubmed/26519555

Jolliffe IT. Principal Component Analysis. Journal of the American Statistical Association. 2002.

Kloppel S, Gregory S, Scheller E, Minkova L, Razi A, Durr A, et al. Compensation in Preclinical Huntington's Disease: Evidence From the Track-On HD Study. EBioMedicine [Internet]. 2015;2:1420-9. Available from: 
http://www.ncbi.nlm.nih.gov/pubmed/26629536

McColgan P, Gregory S, Seunarine KK, Razi A, Papoutsi M, Johnson E, et al. Brain Regions Showing White Matter Loss in Huntington's Disease Are Enriched for Synaptic and Metabolic Genes. Biol Psychiatry. 2018;

Murray LL, Stout JC. Discourse Comprehension in Huntington's and Parkinson's Diseases. Am J Speech-Language Pathol. 2014;

Orth M, Gregory S, Scahill RI, Mayer ISM, Minkova L, Klöppel S, et al. Natural variation in sensory-motor white matter organization influences manifestations of Huntington's disease. Hum Brain Mapp. 2016;37(12):3516-27.

Paulsen JS, Long JD, Ross CA, Harrington DL, Erwin CJ, Williams JK, et al. Prediction of manifest Huntington's disease with clinical and imaging measures: a prospective observational study. Lancet Neurol [Internet]. 2014;13:1193-201. Available from: http://www.ncbi.nlm.nih.gov/pubmed/25453459

Penney JB, Vonsattel JP, MacDonald ME, Gusella JF, Myers RH. CAG repeat number governs the development rate of pathology in huntington's disease. Ann Neurol [Internet]. 1997;41:689-92. Available from: http://www.scopus.com/inward/record.url?eid=2-s2.00030919726\&partnerID=40\&md5=d0160113177d87131ba751bd9e1ac736

Rosas HD, Salat DH, Lee SY, Zaleta AK, Pappu V, Fischl B, et al. Cerebral cortex and the clinical expression of Huntington's disease: complexity and heterogeneity. Brain. 2008;131:1057-68.

Rub U, Seidel K, Vonsattel JP, Lange HW, Eisenmenger W, Gotz M, et al. Huntington's Disease (HD): Neurodegeneration of Brodmann's Primary Visual Area 17 (BA17). Brain Pathol. 2015;25:701-11.

Scahill RI, Hobbs NZ, Say MJ, Bechtel N, Henley SMD, Hyare H, et al. Clinical impairment 
in premanifest and early Huntington's disease is associated with regionally specific atrophy. Hum Brain Mapp. 2013;34:519-29.

Smith SM, Jenkinson M, Woolrich MW, Beckmann CF, Behrens TE, Johansen-Berg H, et al. Advances in functional and structural MR image analysis and implementation as FSL. Neuroimage [Internet]. 2004;23 Suppl 1:S208-19. Available from: http://www.ncbi.nlm.nih.gov/pubmed/15501092

Tabrizi SJ, Langbehn DR, Leavitt BR, Roos RA, Durr A, Craufurd D, et al. Biological and clinical manifestations of Huntington's disease in the longitudinal TRACK-HD study: cross-sectional analysis of baseline data. Lancet Neurol [Internet]. 2009;8:791-801. Available from: http://www.ncbi.nlm.nih.gov/pubmed?term=Biological and clinical manifestations of Huntington\%27s disease in the longitudinal TRACK-HD study

Tabrizi SJ, Reilmann R, Roos RA, Durr A, Leavitt B, Owen G, et al. Potential endpoints for clinical trials in premanifest and early Huntington's disease in the TRACK-HD study: analysis of 24 month observational data. Lancet Neurol [Internet]. 2011/12/06. 2012;11:42-53. Available from: http://www.ncbi.nlm.nih.gov/pubmed/22137354

Tabrizi SJ, Scahill RI, Owen G, Durr A, Leavitt BR, Roos RA, et al. Predictors of phenotypic progression and disease onset in premanifest and early-stage Huntington's disease in the TRACK-HD study: analysis of 36-month observational data. Lancet Neurol [Internet]. 2013;12(7):637-49. Available from: http://www.scopus.com/inward/record.url?eid=2-s2.084877098883\&partnerID=40\&md5=cad80d9ce3f7ce74d8dbce35680a81b1

Thieben MJ, Duggins AJ, Good CD, Gomes L, Mahant N, Richards F, et al. The distribution of structural neuropathology in pre-clinical Huntington's disease. Brain [Internet]. 2002;125:1815-28. Available from: http://www.ncbi.nlm.nih.gov/pubmed/12135972 
Wolf RC, Sambataro F, Vasic N, Baldas EM, Ratheiser I, Bernhard Landwehrmeyer G, et al. Visual system integrity and cognition in early Huntington's disease. Eur J Neurosci [Internet]. 2014;40:2417-26. Available from: http://www.ncbi.nlm.nih.gov/pubmed/24698429

\section{Figure legend}

\section{Figure 1: Multimodal principal component (PC) analysis.}

A. Heat map of correlation coefficients for each modality with dimensions derived from principal component analysis for the combined group of healthy controls and HD participants. The first PC (PC1) showed the highest correlation with structural and functional measures including lower AD and RD in the visual pathway, and thicker V1. The second PC (PC2) showed a pattern of higher FA and lower RD in the visual pathway. The third PC (PC3) captured a pattern of higher RD in the visual pathway. B. Individual participants' PC1 scores differentiated significantly between the control and HD groups $\left({ }^{*} \mathrm{p}=0.026\right)$ while PC2 (C) and PC3 scores (D) were similar in both groups.

Abbreviations: VEP - visual evoked potentials; DTI - Diffusion Tensor Imaging; FA Fractional Anisotropy; AD - Axial Diffusivity; RD - Radial Diffusivity; HD: Huntington's disease. 
Table 1: Demographics and Clinical Measures

\begin{tabular}{|c|c|c|c|c|c|c|}
\hline & \multicolumn{3}{|c|}{ Full sample } & \multicolumn{3}{|c|}{ PCA sample } \\
\hline Variable & $\begin{array}{l}\text { Control } \\
(\mathrm{N}=24)\end{array}$ & HD $(\mathrm{N}=20)$ & $\begin{array}{c}\text { Test Statistic } \\
\text { (p-value) }\end{array}$ & $\begin{array}{l}\text { Control } \\
(\mathrm{N}=20)\end{array}$ & HD ( $N=16)$ & $\begin{array}{c}\text { Test Statistic } \\
\text { (p-value) }\end{array}$ \\
\hline $\begin{array}{l}\text { Gender N } \\
(\% \mathrm{~F})\end{array}$ & $16(66.7)$ & $12(60.0)$ & $\begin{array}{c}\chi^{2}{ }_{1}=0.21 \\
(0.65)\end{array}$ & $15(75.0)$ & $9(56.3)$ & $\begin{array}{c}\chi^{2} 1=1.41 \\
(0.24)\end{array}$ \\
\hline $\begin{array}{l}\text { Age } \\
\text { (SD; Range) }\end{array}$ & $\begin{array}{c}52.5 \\
(9.1 ; 33-68)\end{array}$ & $\begin{array}{c}49.2 \\
(9.6 ; 32-68)\end{array}$ & $\begin{array}{c}t=-1.15 \\
(0.26)\end{array}$ & $\begin{array}{c}53.2 \\
(8.9 ; 33-68)\end{array}$ & $\begin{array}{c}49.4 \\
(9.4 ; 32-68)\end{array}$ & $\begin{array}{c}t=-1.26 \\
(0.22)\end{array}$ \\
\hline Education & $\begin{array}{c}3.7 \\
(1.2 ; 2-6)\end{array}$ & $\begin{array}{c}4.3 \\
(0.9 ; 2-5)\end{array}$ & $\begin{array}{c}\mathrm{t}=1.97 \\
(0.06)\end{array}$ & $\begin{array}{c}3.6 \\
(1.3 ; 2-6)\end{array}$ & $\begin{array}{c}4.5 \\
(0.7 ; 3-5)\end{array}$ & $\begin{array}{c}\mathrm{t}=2.80 \\
(0.01)\end{array}$ \\
\hline $\begin{array}{l}\text { CAG Repeat } \\
\text { Length } \\
\text { (SD; Range) }\end{array}$ & -- & $\begin{array}{c}42.8 \\
(3.0 ; 39-50)\end{array}$ & -- & -- & $\begin{array}{c}42.5 \\
(2.9 ; 39-50)\end{array}$ & -- \\
\hline $\begin{array}{l}\text { Disease } \\
\text { Burden } \\
\text { (SD; Range) }\end{array}$ & -- & $\begin{array}{c}338 \\
(79 ; 192-478)\end{array}$ & -- & -- & $\begin{array}{c}326 \\
(79 ; 192-469)\end{array}$ & -- \\
\hline $\begin{array}{l}\text { Motor score } \\
\text { (SD; Range) }\end{array}$ & $\begin{array}{c}1.3 \\
(1.7 ; 0-5)\end{array}$ & $\begin{array}{c}12.8 \\
(11.4 ; 1-50)\end{array}$ & $\begin{array}{c}t=4.48 \\
(0.0002)\end{array}$ & $\begin{array}{c}1.2 \\
(1.7 ; 0-5)\end{array}$ & $\begin{array}{c}10.1 \\
(7.6 ; 1-28)\end{array}$ & $\begin{array}{c}t=4.59 \\
(0.0003)\end{array}$ \\
\hline
\end{tabular}


Table 2: Individual Modality Results

\begin{tabular}{lccccccccc} 
Modality & & Controls & \multicolumn{2}{c}{ HD } & & & & & \\
& & Mean & SD & Mean & SD & t-test & df & p-value & q-value \\
\hline CT & V1 & 3853 & 665 & 3713 & 513 & 0.77 & 42 & 0.45 & 0.45 \\
\hline DTI & FA & 0.35 & 0.034 & 0.32 & 0.056 & 1.78 & 23.1 & 0.089 & 0.24 \\
& AD & 1.14 & 0.051 & 1.16 & 0.092 & -0.85 & 22.0 & 0.44 & 0.45 \\
& RD & $\mathbf{0 . 6 5}$ & $\mathbf{0 . 0 3 9}$ & $\mathbf{0 . 7 2}$ & $\mathbf{0 . 0 6 5}$ & $\mathbf{- 2 . 8 6}$ & $\mathbf{2 3 . 0}$ & $\mathbf{0 . 0 1 4}$ & $\mathbf{0 . 1 1}$ \\
\hline VEP P100 & Latency & 100.1 & 6.0 & 103.3 & 6.0 & -1.78 & 42 & 0.082 & 0.24 \\
& Amplitude & 6.72 & 2.43 & 5.87 & 2.36 & 1.16 & 41 & 0.25 & 0.33 \\
\hline Behaviour & Map & 65.63 & 8.09 & 62.40 & 9.12 & 1.24 & 42 & 0.22 & 0.33 \\
& Search & & & & & & & & \\
& SDMT & 55.42 & 11.87 & 50.16 & 10.53 & 1.52 & 41 & 0.14 & 0.27 \\
\hline
\end{tabular}

Descriptive statistics and group comparison data for each MRI, electrophysiological and behavioural measure. Abbreviations: SD - standard deviation; df - degrees of freedom; q-value: false discovery rate adjusted p-value; CT- Cortical Thickness; DTI- Diffusion tensor Imaging; VEP - Visual Evoked Potentials; V1 - Primary Visual Cortex; 01 - left occipital region; FA - Fractional Anisotropy; RD - Radial Diffusivity; AD - Axial Diffusivity 
Table 3: Correlations Between Two Behaviour Measures and 6 Structure-Function Measures for HD Participants

\begin{tabular}{|c|c|c|}
\hline Modality & Map Search & SDMT \\
\hline \multirow[t]{2}{*}{ FA } & $r=-0.34(p=0.20 ; q=0.47)$ & $r=0.63(p=0.0091 ; q=0.055)$ \\
\hline & 16 & 16 \\
\hline \multirow[t]{2}{*}{ AD } & $r=-0.16(p=0.56 ; q=0.61)$ & $r=0.10(p=0.72 ; q=0.72)$ \\
\hline & 16 & 16 \\
\hline \multirow[t]{2}{*}{ RD } & $\mathrm{r}=0.23(\mathrm{p}=0.40 ; \mathrm{q}=0.60)$ & $r=-0.55(p=0.026 ; q=0.11)$ \\
\hline & 16 & 16 \\
\hline V1 Cortical & $r=0.25(p=0.30 ; q=0.59)$ & $r=0.16(p=0.50 ; q=0.61)$ \\
\hline Thickness & 20 & 19 \\
\hline VEP P100 & $r=-0.60(p=0.0056 ; q=0.055)$ & $r=-0.41(p=0.079 ; q=0.24)$ \\
\hline Latency & 20 & 19 \\
\hline VEP P100 & $r=-0.15(p=0.52 ; q=0.61)$ & $r=0.22(p=0.36 ; q=0.60)$ \\
\hline Amplitude & 20 & 19 \\
\hline
\end{tabular}

Pearson's correlations ( $\mathrm{p}$-value; q-value) and sample size for each MRI, electrophysiological and behavioural measure. Abbreviations: V1 - Primary Visual Cortex; 01 - left occipital region; FA - Fractional Anisotropy; RD - Radial Diffusivity; AD - Axial Diffusivity 
Table 4: Correlations Between Two Behaviour Measures and 6 Structure-Function Measures for Control Participants

\begin{tabular}{|c|c|c|}
\hline Modality & Map Search & SDMT \\
\hline \multirow[t]{2}{*}{ FA } & $r=-0.092(p=0.69 ; q=0.86)$ & $r=-0.079(p=0.73 ; q=0.86)$ \\
\hline & 21 & 21 \\
\hline \multirow[t]{2}{*}{ AD } & $r=-0.077(p=0.74 ; q=0.86)$ & $\mathrm{r}=0.0080(\mathrm{p}=0.97 ; \mathrm{q}=0.97)$ \\
\hline & 21 & 21 \\
\hline \multirow[t]{2}{*}{ RD } & $\mathrm{r}=0.15(\mathrm{p}=0.53 ; \mathrm{q}=0.86)$ & $r=0.064(p=0.78 ; q=0.86)$ \\
\hline & 21 & 21 \\
\hline \multirow{2}{*}{$\begin{array}{l}\text { V1 Cortical } \\
\text { Thickness }\end{array}$} & $r=0.13(p=0.53 ; q=0.86)$ & $r=-0.27(p=0.20 ; q=0.86)$ \\
\hline & 24 & 24 \\
\hline \multirow{2}{*}{$\begin{array}{l}\text { VEP P100 } \\
\text { Latency }\end{array}$} & $r=-0.15(p=0.48 ; q=0.86)$ & $\mathrm{r}=-0.13(\mathrm{p}=0.53 ; \mathrm{q}=0.86)$ \\
\hline & 24 & 24 \\
\hline \multirow{2}{*}{$\begin{array}{l}\text { VEP P100 } \\
\text { Amplitude }\end{array}$} & $r=-0.27(p=0.21 ; q=0.86)$ & $r=-0.11(p=0.62 ; q=0.86)$ \\
\hline & 23 & 23 \\
\hline
\end{tabular}

Pearson's correlations (p-value; q-value) and sample size for each MRI, electrophysiological and behavioural measure. Abbreviations: V1 - Primary Visual Cortex; 01 - left occipital region; FA - Fractional Anisotropy; RD - Radial Diffusivity; AD - Axial Diffusivity 
Table 5: Correlations Between 3 Classic Principal Components and 6 StructureFunction Measures

\begin{tabular}{|l|r|r|r|}
\hline & Comp1 & Comp2 & Comp3 \\
\hline FA & $\mathbf{0 . 6 3}$ & $\mathbf{- 0 . 7 6}$ & -0.11 \\
\hline AD & -0.38 & $\mathbf{- 0 . 7 1}$ & $\mathbf{- 0 . 5 0}$ \\
\hline RD & $\mathbf{- 0 . 8 9}$ & 0.21 & -0.28 \\
\hline V1 Cortical & 0.36 & $\mathbf{0 . 4 1}$ & $\mathbf{- 0 . 7 2}$ \\
Thickness & & & \\
\hline VEP P100 Latency & $\mathbf{- 0 . 4 1}$ & -0.089 & -0.23 \\
\hline $\begin{array}{l}\text { VEP P100 } \\
\text { Amplitude }\end{array}$ & $\mathbf{0 . 6 4}$ & 0.33 & -0.32 \\
\hline
\end{tabular}

Abbreviations: V1 - Primary Visual Cortex; 01 - left occipital region; FA - Fractional Anisotropy; RD - Radial Diffusivity; AD - Axial Diffusivity 